\title{
Investigation of the Intestinal Permeability and First-Pass Metabolism of Drugs in Cynomolgus Monkeys Using Single-Pass Intestinal Perfusion
}

\author{
Masayuki Takahashi, ${ }^{* a}$ Takuo Washio, ${ }^{b}$ Norio Suzuki, ${ }^{a}$ Katsuhiro Igeta, ${ }^{a}$ and Shinji Yamashita ${ }^{c}$ \\ ${ }^{a}$ Drug Metabolism and Pharmacokinetics Research Laboratories, Daiichi-Sankyo Co., Ltd.; ${ }^{b}$ Asia Development \\ Department, Daiichi-Sankyo Co., Ltd.; 1-2-58 Hiromachi, Shinagawa-ku, Tokyo 140-8710, Japan: and ${ }^{c}$ Faculty of \\ Pharmaceutical Sciences, Setsunan University; 45-1 Nagaotoge-cho, Hirakata, Osaka 573-0101, Japan. \\ Received August 20, 2009; accepted October 7, 2009; published online October 15, 2009
}

To clarify the causes of low oral bioavailability (BA) of drugs in cynomolgus monkeys, the experimental method to evaluate the drug permeability and the metabolism in the intestine of cynomolgus monkeys was established. An in situ intestinal perfusion method was performed with blood sampling from both portal and peripheral veins to calculate the intestinal permeability and the metabolism of drugs simultaneously. In all experiments, antipyrine was co-perfused with test drugs as a non-metabolized reference to calculate the individual portal vein blood flow. The effective permeability coefficient $\left(P_{\text {eff }}\right)$ of acetaminophen and piroxicam were high, and the fraction of dose absorbed from the gastrointestinal tract (Fa) thought to be 1. The intestinal availability (Fg) of acetaminophen and piroxicam were calculated to be 0.39 and 1.09 , respectively. The Fa*Fg values of these drugs calculated from the perfusion study almost coincided with those obtained from the in vivo PK analysis in the previous report. In addition, the Fg values of verapamil and midazolam were calculated as 0.16 and 0.26 , respectively, suggesting these drugs were metabolized extensively in the intestine after oral administration to cynomolgus monkey. Furthermore, the Fg values of these drugs were increased to $0.8-0.85$ in the presence of 1 -aminobenzotriazole, a typical cytochrome P450 (CYP) inhibitor. In conclusion, it was clarified that acetaminophen, verapamil and midazolam were metabolized extensively in the intestine of cynomolgus monkeys. This intestinal perfusion method is considered to be useful to identify the factors of species difference in the oral absorption of drugs.

Key words intestinal absorption; first-pass metabolism; cynomolgus monkey; intestinal perfusion; bioavailability

Pharmacokinetic (PK) data in experimental animals are important for the profiling and selection of candidate compounds in the drug discovery process. In particular, oral bioavailability (BA) is one of the most important factors for the quality of new oral chemical entities. Oral BA of drugs is defined by the following equation as,

$\mathrm{BA}=\mathrm{Fa} * \mathrm{Fg} * \mathrm{Fh}$

where $\mathrm{Fa}$ is the fraction of dose absorbed from the gastrointestinal (GI) tract, Fg is the intestinal availability, that correspondent to the fraction of dose not metabolized in the intestinal wall during the absorption process and $\mathrm{Fh}$ is the fraction of dose not metabolized in the liver before reaching the systemic circulation. Therefore, the values of $(1-\mathrm{Fg})$ and $(1-\mathrm{Fh})$ mean the first-pass metabolism of orally administered drugs in GI tract and the liver, respectively. In order to select the appropriate candidates for oral drug product, quantitative assessments on each process are highly required.

Recently, not only the small animals such as mice and rats, but also cynomolgus monkeys are often used as an animal model for PK studies in the drug discovery stage. However, lower oral BAs of test compounds were often observed in cynomolgus monkeys compared with those in other animals. ${ }^{1,2}$ Also in our previous study, various marketed drugs showed low oral BAs in cynomolgus monkeys than those in humans. From the kinetic analysis of the plasma concentrations of drugs after intravenous and oral administration, it was indicated that the low oral BAs in cynomolgus monkeys are mostly attributed to the low value of Fa*Fg. ${ }^{3,4)}$ However, only from the in vivo PK analysis, it is theoretically impossible to evaluate Fa and Fg separately, resulting the confusion in the contribution of intestinal absorption on the oral BA of various drugs.

Tamura et al. investigated the mechanisms of intestinal absorption of tacrolims by using the simultaneous perfusion method of intestinal lumen and mesenteric veins in rats. ${ }^{5}$ They have calculated the fraction of metabolized in the intestinal cells from the difference between eliminated amount of the drug from the intestinal lumen and absorbed amount into the blood perfusate. Also, Mihara et al. evaluated quantitatively the first-pass metabolism of eperison in rats by using the similar perfusion method. ${ }^{6}$ ) Although the simultaneous perfusion method is useful to assess the intestinal metabolism of drugs, this method requires complicated operations of the mesenteric vein and is difficult to perform in cynomolgus monkeys. Moreover, perfusion of the mesenteric vein might affect the absorption and metabolism of drugs in the intestine.

The present study is performed 1) to establish the experimental method to evaluate the drug permeability and the metabolism in the intestine of cynomolgus monkeys and then 2) to clarify the causes of low BA of drugs in cynomolgus monkeys. For these purposes, an in situ single-pass intestinal perfusion method was performed with blood sampling from both portal and peripheral veins, instead of the perfusion of the mesenteric vein. The intestinal permeability of drugs $\left(P_{\text {eff }}\right)$ is calculated from the decrease in drug concentration in the perfusate after single pass perfusion through the intestinal tract. The intestinal metabolism during the absorption process $(1-\mathrm{Fg})$ is calculated from the difference between drug amounts that disappeared from the perfusate and absorbed into the portal vein as intact. In this method, the amount of absorbed drugs into the portal vein was calculated by multiplying the portal vein blood flow ( $\left.V_{\text {portal }}\right)$ to the drug 
concentration which was obtained by subtracting the peripheral blood concentration from the portal vein blood concentration. Since $V_{\text {portal }}$ is considered to deviate extensively due to the effect of anesthesia, in our method, the value of $V_{\text {portal }}$ in each monkey was calculated from the result with non-metabolized reference drug. Antipyrine was used as a reference drug and co-perfused with all other drugs since its $\mathrm{Fa} * \mathrm{Fg}$ value was reported to be 1 in cynomolgus monkeys. ${ }^{4)}$ In order to validate this method, the effect of 1-aminobenzotriazole (ABT), a typical cytochrome P450 (CYP) inhibitor, on the Fg values of CYP-substrate drugs was investigated.

This study is expected to provide a new experimental method that enables the quantitative evaluation of the intestinal metabolisms in large animals such as monkeys and dogs and to clarify the factors of species difference in oral drug absorption.

\section{MATERIALS AND METHODS}

Materials Acetaminophen, propranolol, atenolol, antipyrine, piroxicam, verapamil, midazolam and 1-aminobenzotriazole (ABT) were purchased from Sigma-Aldrich (St. Louis, MO, U.S.A.). D82-7319, an internal standard for the LC/MS/MS analysis was synthesized by the Medicinal Chemistry Research Laboratory, Daiichi Pharmaceutical Co., Ltd. (Tokyo, Japan). All other chemicals were obtained from commercial sources and were of analytical or the highest grade available.

Animals Male cynomolgus monkeys, weighing 3.0$5.7 \mathrm{~kg}$, were used. The perfusion experiments were carried out at Primate Ltd. (Guangdong Province, China). The cynomolgus monkeys were fasted overnight prior to the perfusion study, whereas access to water was provided ad libitum. In the intestinal perfusion studies, 12 monkeys were divided into 4 groups.

Preparation of Perfusate Antipyrine was added to the every perfusate to calculate the $V_{\text {portal }}$. Other two or three drugs were also added simultaneously as one cassette in each group. The drugs were dissolved completely (final concentration: $10 \mu \mathrm{M}$ ) in phosphate buffered saline (PBS) and the $\mathrm{pH}$ was adjusted to 6.5 with $1 \mathrm{~N} \mathrm{HCl}$ or $1 \mathrm{~N} \mathrm{NaOH}$. Additionally, propranolol, verapamil and midazolam were perfused in the presence and absence of $100 \mu \mathrm{M}$ ABT.

In Situ Intestinal Perfusion Experiment The cynomol- gus monkeys were anesthetized with ketamine (about $0.5 \mathrm{mg} / \mathrm{kg}$, intramuscularly) before the perfusion experiments. Under anesthesia, an incision was made in the abdomens of the monkeys and inner tube connectors (i.d. 5$8 \mathrm{~mm}$ ) were inserted to the perfusion part in both the upper part (inlet) and the lower part (outlet). The length of the perfusion was set at about $20 \mathrm{~cm}$ in the upper part of the small intestine. The perfusate and PBS ( $\mathrm{pH} \mathrm{6.5)} \mathrm{were} \mathrm{put} \mathrm{into} \mathrm{a}$ water bath $\left(37^{\circ} \mathrm{C}\right)$ and kept warm. The micro-tube pump was connected to the inner tube connector and the flow was started. After flushing three times with PBS ( $\mathrm{pH}$ 6.5) and once with perfusate, the flow rate of the perfusate was adjusted to $1.5-2.0 \mathrm{ml} / \mathrm{min}$. The flow rate (FR) was precisely calculated by measuring weight of collected perfusate from outlet tube. The perfusate was passed for $30 \mathrm{~min}$ in advance to equalize the flow rate and the temperature in the inner tube. After equalizing, blood was taken from the peripheral and portal veins every $20 \mathrm{~min}$ and the perfusate was collected every $10 \mathrm{~min}$ for $1.5 \mathrm{~h}$. After perfusion, each circumference and length of the perfused intestine was measured. The scheme of the intestinal perfusion method in cynomolgus monkeys is shown in Fig. 1.

All the animal experiments were conducted with the approval of the Animal Experiment Ethics Committee of Daiichi Pharmaceutical Co., Ltd. and Primate Inc.

LC/MS/MS Analysis The concentrations of the tested drugs in the perfusate, peripheral blood and portal vein blood were assayed by an LC/MS/MS method consisting of an Alliance 2795 (Waters, Milford, MA, U.S.A.) and a TSQ7000 quadrupole mass spectrometer (ThermoFisher, Waltham, MA, U.S.A.). Multiple reaction monitoring (MRM) mode was used. An Atlantis dC18 column $(3 \mu \mathrm{m} \phi, 2.1 \mathrm{~mm} \times$ $20 \mathrm{~mm}$, Waters) warmed to $40{ }^{\circ} \mathrm{C}$ was used for separation. The elution was carried out at a flow rate of $0.5 \mathrm{ml} / \mathrm{min}$ by a linear gradient with the mobile phase, which consisted of a mixture of $\mathrm{A}$ ( $10 \mathrm{~mm}$ ammonium formate solution) and $\mathrm{B}$ (Acetonitrile). The gradient conditions for elution were as follows: Gradient: $[\mathrm{min}, \mathrm{B} \%]=[0,30]-[0.5,30]-[1.5,90]-$ $[3.5,90]-[4,30]-[5,30]$.

Sample Measurement Each perfusate was diluted 10 times by PBS ( $\mathrm{pH}$ 6.5). Aliquot of the diluted perfusate and plasma samples $(50 \mu \mathrm{l})$ were transferred separately to the wells of a 96-well plate, and IS solution $(100 \mu 1,100 \mathrm{ng} / \mathrm{ml}$ of acetonitrile solution) was added to each sample. The plate

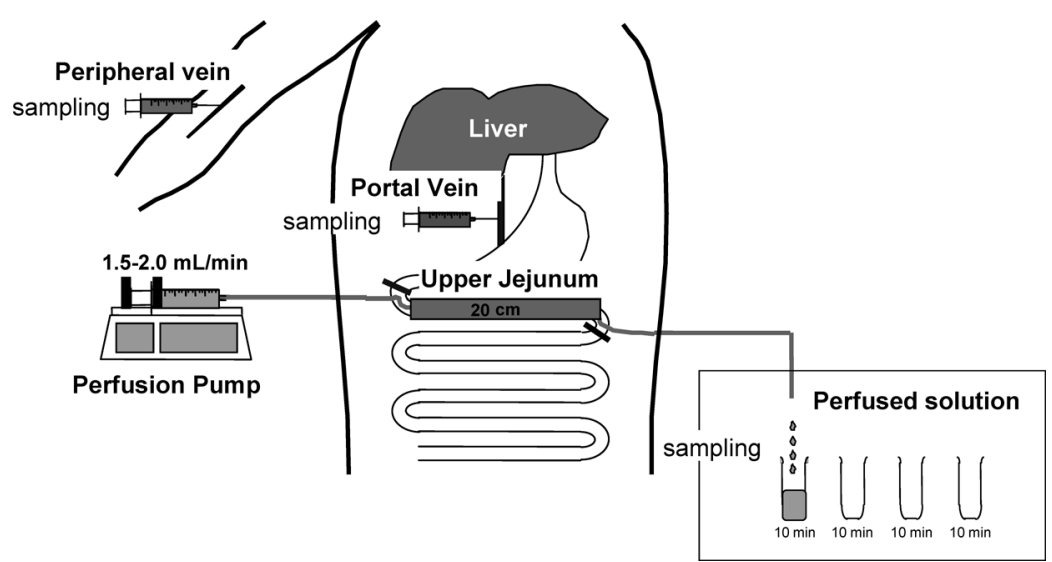

Fig. 1. Scheme of the Intestinal Perfusion Method in Cynomolgus Monkeys 


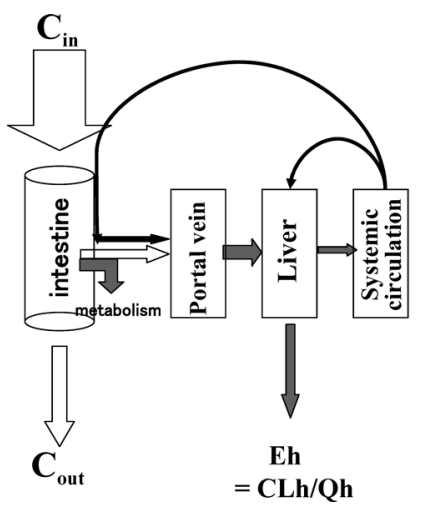

$\mathbf{P}_{\mathrm{eff}}=\mathbf{C L}_{\mathrm{ab}} /$ (circumference $\times$ length of intestine )

$\mathrm{CL}_{\mathrm{ab}}=\left(\mathrm{C}_{\mathrm{in}}-\mathbf{C}_{\text {out }}\right) \times \mathbf{F R} / \mathbf{C}_{\mathrm{in}}$

$\mathbf{F g}=\mathrm{C}_{\text {abs }} \times \mathrm{V}_{\text {portal }} /\left(\mathrm{C}_{\text {in }}-\mathrm{C}_{\text {out }}\right) \times \mathbf{F R}$

$\mathrm{C}_{\text {abs }}=\left(\mathrm{C}_{\text {portal }}-\mathrm{C}_{\text {peri }}\right) \times \mathrm{R}_{\mathrm{b}}$

$V_{\text {portal }}=\left(C_{\text {in }}-C_{\text {out }}\right) \times F R / C_{a b s}$ of Antipyrine*

$*:$ Fg of Antipyrine $=1$

Fig. 2. Theory of PK Analysis for Intestinal Perfusion Methods in Cynomolgus Monkeys

was then centrifuged at $1800 \mathrm{~g}$ for $10 \mathrm{~min}$ at $4{ }^{\circ} \mathrm{C}$. The supernatant was transferred to a MultiScreen (GV, $0.22 \mu \mathrm{m}$, Millipore, Billerica, MA, U.S.A.) and filtered by centrifugation at $1800 \mathrm{~g}$ for $10 \mathrm{~min}$ at $4{ }^{\circ} \mathrm{C}$. The filtrate was transferred to auto-sampler vials and a $10 \mu \mathrm{l}$ portion was injected onto the LC/MS/MS system. Calibration standard curves for each perfusate and plasma sample were made at concentrations of $1,5,10,50,100,500,1000,5000 \mathrm{ng} / \mathrm{ml}$. The peak area of the drugs was divided by the peak area of the IS to obtain the peak-area ratio. The calibration curve for the drug was constructed from a least-square linear regression of the peakarea ratios of the standards versus the drug concentrations.

Kinetic Analysis In this study, the absorption clearance from the perfused intestine $\left(C L_{\mathrm{ab}}\right)$ was calculated from the drug concentration in the perfusate just before perfusion $\left(C_{\text {in }}\right)$, the drug concentration in the perfusate after perfusion $\left(C_{\text {out }}\right)$ and the perfusion rate (FR), according to Eq. 2. $P_{\text {eff }}$ of each drug was calculated by dividing $C L_{\mathrm{ab}}$ by the surface area of the perfused intestine (the inner circumference $X$ length of the intestinal tract) as shown in Eq. 3.

$$
\begin{aligned}
& C L_{\mathrm{ab}}=\left(C_{\mathrm{in}}-C_{\text {out }}\right) \times \mathrm{FR} / C_{\mathrm{in}} \\
& P_{\text {eff }}=C L_{\mathrm{ab}} /(\text { circumference } \times \text { length of intestine })
\end{aligned}
$$

The Fg values of each drug were calculated by the ratio of the amount of drugs $\left(\left(C_{\text {in }}-C_{\text {out }}\right) \times \mathrm{FR} \times\right.$ time $)$ which had disappeared from the perfusate in the small intestine and the amount of drugs which had been absorbed into the portal vein (the absorbed drug concentration in the portal vein $\left(C_{\mathrm{abs}}\right) \times V_{\text {portal }} \times$ time $)$, according to Eq. 4 . In this calculation, the $C_{\text {abs }}$ was calculated by subtracting the peripheral blood concentration $\left(C_{\text {peri }}\right)$ from the portal vein blood concentration $\left(C_{\text {portal }}\right)$, as shown in Eq. 5. The blood-to-plasma concentration ratio $\left(R_{\mathrm{b}}\right)$ values of the tested drugs were determined in the previous study. ${ }^{3,4)}$ However, since it was difficult to determine $V_{\text {portal }}$, it was calculated from the $\left(C_{\text {in }}-C_{\text {out }}\right)$ and $C_{\text {abs }}$ of antipyrine, having assumed the $\mathrm{Fg}$ of antipyrine to be 1 , as shown in Eq. 6. The theory regarding the PK analysis for intestinal perfusion methods in cynomolgus monkeys is summarized in Fig. 2.

$$
\begin{aligned}
& \mathrm{Fg}=C_{\text {abs }} \times V_{\text {portal }} /\left(C_{\text {in }}-C_{\text {out }}\right) \times \mathrm{FR} \\
& C_{\text {abs }}=\left(C_{\text {portal }}-C_{\text {peri }}\right) \times R_{\mathrm{b}} \\
& V_{\text {portal }}=\left(C_{\text {in }}-C_{\text {out }}\right) \times \mathrm{FR} / C_{\text {abs }} \text { of antipyrine* } \\
& *: \text { Fg of antipyrine }=1
\end{aligned}
$$

Table 1. $P_{\text {eff }}$ Values of the Test Drugs Evaluated Using the Intestinal Perfusion Method in Cynomolgus Monkeys

\begin{tabular}{lcc}
\hline \hline \multirow{2}{*}{ Drugs } & \multicolumn{2}{c}{$P_{\text {eff }}\left(\times 10^{-4} \mathrm{~cm} / \mathrm{s}\right)$} \\
\cline { 2 - 3 } & \multicolumn{1}{c}{ Cynomolgus monkeys } & Humans $^{a)}$ \\
\hline Antipyrine & $1.36 \pm 0.46$ & 5.6 \\
Atenolol & $0.37 \pm 0.12$ & 0.2 \\
Acetaminophen & $1.57 \pm 0.31$ & - \\
Piroxicam & $2.24 \pm 0.56$ & 6.65 \\
Propranolol & $1.05 \pm 0.40$ & 2.91 \\
Verapamil & $0.93 \pm 0.64$ & 6.8 \\
Midazolam & $1.74 \pm 0.60$ & -
\end{tabular}

a) Human data are cited from the literature. ${ }^{7}$

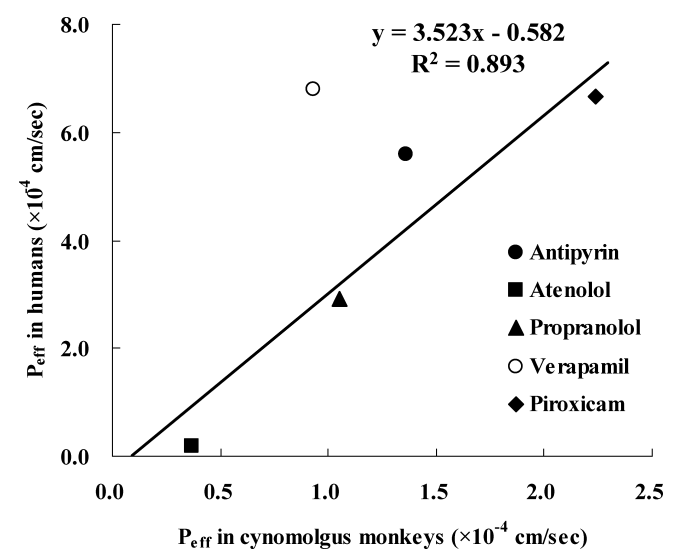

Fig. 3. Relationship of the $P_{\text {eff }}$ of the Test Drugs between Cynomolgus Monkeys and Humans

The solid line represents the correlation curve for the $P_{\text {eff }}$ values between cynomolgus monkeys and humans with the exception of verapamil. Human data are cited from the literature. ${ }^{7}$

\section{RESULTS}

$\boldsymbol{P}_{\text {eff }}$ of Test Drugs in Cynomolgus Monkeys $P_{\text {eff }}$ to the intestinal membrane of cynomolgus monkeys was evaluated from the difference of drug concentrations in the perfusate before and after the perfusion (Eqs. 2, 3). The $P_{\text {eff }}$ values of the test drugs in cynomolgus monkeys were shown in Table 1 with their $P_{\text {eff }}$ values in humans taken from the literature. ${ }^{7)}$ In addition, the relationship of the $P_{\text {eff }}$ values of test drugs between cynomolgus monkeys and humans was shown in Fig. 3. Except for verapamil, the $P_{\text {eff }}$ of test drugs in cynomolgus monkeys showed good correlation with those in humans $\left(R^{2}=0.893\right)$. However, the absolute values of $P_{\text {eff }}$ were lower 
Table 2. Fg Values of the Test Drugs Calculated Using the Intestinal Perfusion Method in Cynomolgus Monkeys

\begin{tabular}{lccl}
\hline \hline \multirow{2}{*}{ Drug } & \multirow{2}{*}{$\begin{array}{c}\text { Fg in } \\
\text { cynomolgus } \\
\text { monkeys }\end{array}$} & \multicolumn{2}{c}{ In vivo Fa*Fg (bioavailability) } \\
\cline { 3 - 4 } & & Cynomolgus monkeys & Humans \\
\hline Antipyrine & 1 & $0.99(86 \%)$ & $1(100 \%)$ \\
Atenolol & n.c. & $0.58(57 \%)$ & $0.59(58 \%)$ \\
Acetaminophen & $0.39 \pm 0.21$ & $0.19(16 \%)$ & $1(88 \%)$ \\
Piroxicam & $1.09 \pm 0.01$ & $0.96(95 \%)$ & $1(100 \%)$ \\
Propranolol & $0.66 \pm 0.03$ & n.c. $(<1 \%)$ & $1(26 \%)$ \\
Verapamil & $0.16 \pm 0.09$ & n.c. $(<1 \%)$ & $0.71(22 \%)$ \\
Midazolam & $0.26 \pm 0.13$ & n.c. $(<1 \%)$ & $0.64(44 \%)$ \\
& & & \\
\hline
\end{tabular}

n.c.: not calculated. Fa*Fg values in cynomolgus monkeys represents the mean of the data. Data are cited from the previous report. ${ }^{4)}$

in cynomolgus monkeys than in humans for most of drugs. Only atenolol showed the similar value of $P_{\text {eff }}$ between cynomolgus monkeys and humans, suggesting the comparable permeability for paracellular but the lower permeability for transcellular pathway in the intestinal membrane of cynomolgus monkeys. However, since we have already reported that antipyrine was completely absorbed in vivo, ${ }^{4)}$ the $P_{\text {eff }}$ values of acetaminophen, antipyrine, piroxicam and midazolam might confirm that these drugs are absorbed almost completely in cynomolgus monkeys after oral administration (i.e. $\mathrm{Fa}=1$ ).

Fg of Test Drugs Calculated from the Intestinal Perfusion Method in Cynomolgus Monkeys Intestinal firstpass metabolism of test drugs were evaluated from both the intestinal perfusion data and the blood concentration data. The Fg values were calculated according to Eqs. 4-6 and shown in Table 2 with the Fa*Fg values of drugs obtained from in vivo PK analysis in the previous report. ${ }^{4}$ In this study, the Fg value of atenolol could not be calculated because significant differences in the concentration between portal vein and peripheral vein were not detected due to the low concentrations of atenolol in both veins. Since high $P_{\text {eff }}$ values of acetaminophen, antipyrine, piroxicam and midazolam indicated the complete absorption in vivo $(\mathrm{Fa}=1), \mathrm{Fa} * \mathrm{Fg}$ values of these drugs can be regarded as almost equal to Fg. Then, in both studies, piroxicam was revealed not to be metabolized in the intestine ( $\mathrm{Fg}$ is approximately 1). The $\mathrm{Fg}$ value of acetaminophen was calculated as $0.39 \pm 0.21$ in this study that is 2-times higher than the in vivo estimation. However, extraction ratios in the intestine $(1-\mathrm{Fg})$ of acetaminophen in the in situ and in vivo experiments can be calculated to be 0.61 and 0.81 , respectively. Both data have demonstrated the major impact of intestinal metabolism on the oral absorption of acetaminophen in cynomolgus monkeys, but not in humans. In the case of propranolol and verapamil, although exact values of $\mathrm{Fa}^{*} \mathrm{Fg}$ could not be calculated in vivo due to the very low oral absorption (BA was less than $1 \%$ ), in situ perfusion study clearly showed the differences in $\mathrm{Fg}$ values of both drugs. Verapamil, a typical substrate of CYP3A enzyme, was extensively metabolized in the intestine of cynomolgus monkeys whereas propranolol, a substrate of CYP2D6, was metabolized only 30-40\%. Another CYP3A substrate drug, midazolam, also showed the very low value of Fg, suggesting the high activity of CYP3A enzyme in the intestine of cynomolgus monkeys.

Effects of ABT on Fg of Midazolam, Propranolol and

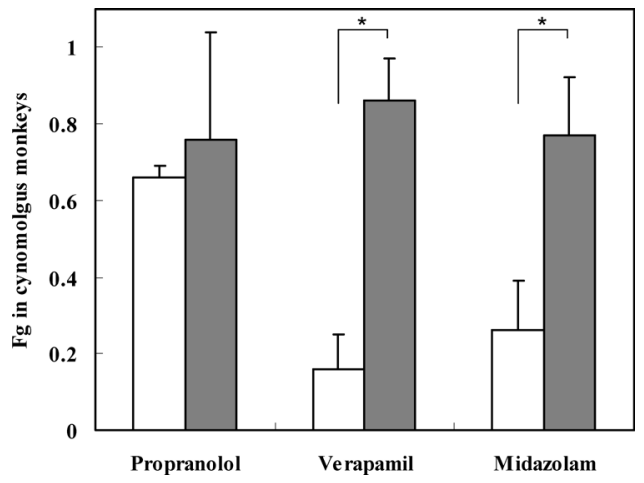

Fig. 4. Fg Values of Propranolol, Verapamil and Midazolam in the Presence (Open Bars) or Absence (Closed Bars) of ABT Calculated Using the in Situ Intestinal Perfusion Method in Cynomolgus Monkeys

Results are expressed as the mean \pm S.D. $(n=3)$. * The difference was significant compared with the control $(p<0.05)$ by a Student's $t$-test.

Verapamil The Fg values of propranolol, verapamil and midazolam were assessed in the presence of ABT, a non-specific inhibitor of CYP enzymes, as shown in Fig. 4. ABT remarkably enhanced the Fg values of verapamil and midazolam, a typical substrate of CYP3A enzyme, but that of propranolol increased only slightly. These results well confirmed that the oral absorption of verapamil and midazolam in cynomolgus monkeys was strongly regulated by the high activity of CYP3A enzymes in the intestine. Also, the results in Fig. 4 can validate the experimental methods and the theory to evaluate the Fg values of the drugs used in this perfusion study since Fg values in the presence of ABT were calculated about 0.8 for all three drugs.

\section{DISCUSSION}

In this study, we have evaluated both intestinal permeability and metabolism of drugs in cynomolgus monkeys to consider the cause of their low oral BA observed in the previous report. ${ }^{3,4)}$ In order to assess the permeability of drugs to the intestinal membrane, various in vitro, in situ and in vivo methods have been utilized. ${ }^{89}$ ) Recently, in vitro cell culture models with Caco- $2^{10-13)}$ or Madin-Darby canine kidney $(\mathrm{MDCK})^{14,15)}$ cells have been widely used to measure the membrane permeability of drugs. Furthermore, a parallel artificial membrane permeability assay system (PAMPA) has been developed as a high throughput screening of drug permeability. ${ }^{16,17)}$ On the other hand, in vivo permeability of various drugs to the human intestine has been evaluated by intubation method and used to analyze the relation between intestinal permeability of drugs and their oral absorption. ${ }^{18-20)}$ However, in situ or in vivo drug permeability in the monkey intestine has not been reported yet because in situ intestinal perfusion method is usually applied to the small animals such as rat. ${ }^{5,6,21,22)}$ In order to consider the differences in the intestinal drug absorption between cynomolgus monkeys and humans, the same quality of permeability data should be necessary for both species.

In the perfusion study, the drug concentration in the perfusate and the perfusion region were important to consider the in vivo absorption of test drugs. In this study, the drug concentration in the perfusate was unified to $10 \mu \mathrm{M}$ to avoid the saturation of the metabolic enzymes and the transporters. 
Then, results were compared mainly with the in vivo absorption in our previous reports in which the low doses of drugs were orally administered to cynomolgus monkeys to observe the non-saturated absorption kinetics. Concerning the sitedependent differences in drug permeability, upper part of intestine is generally considered to have the higher permeability than the lower part. ${ }^{23)}$ However, in the most of reports, proximal jejunum were used for perfusion study to measure $P_{\text {eff }}$ because the main absorption site of drugs is proximal intestine. ${ }^{18-20)}$ In addition, Paine et al. demonstrated that the upper small intestine is the major site for CYP3A-mediated first-pass metabolism in humans. ${ }^{24)}$ Therefore, the intestinal perfusion in this study was carried out using upper part of the small intestine.

As shown in Table 1 and Fig. 3, except for verapamil, the $P_{\text {eff }}$ values of test drugs in cynomolgus monkeys showed a good correlation with those in humans $\left(R^{2}=0.893\right)$. However, absolute values of $P_{\text {eff }}$ of highly permeable drugs (antipyrine, piroxicam and propranolol) were 3-4 times lower in cynomolgus monkeys than in humans, whereas $P_{\text {eff }}$ value of atenolol was almost comparable. Although the reason of this difference in the intestinal permeability of drugs is unclear, this result might emerge the low absorbability of cynomolgus monkeys for highly permeable drugs that dominantly permeate through the transcellular pathway of the intestinal membrane. However, Fagerholm et al. have reported that the fraction dose absorbed of drugs from the intestinal tract (Fa) can be expressed as

$$
\mathrm{Fa}=1-\exp \left(-(2 / R) \times P_{\text {eff }} \times t_{\text {tran }}\right)
$$

where $R$ is the radius of the intestinal tract and $t_{\text {tran }}$ is the intestinal transit time of the drug. ${ }^{18)}$ Since we have already demonstrated that the $t_{\text {tran }}$ of cynomolgus monkeys are almost comparable with that of humans, the value of $P_{\text {eff }} / R$ should be crucial for considering the difference in drug absorbability. A radius of the small intestine of cynomolgus monkeys used in this study was in the range of $0.35-0.45 \mathrm{~cm}$ while that of human is reported to be around $2.5 \mathrm{~cm} .{ }^{25)}$ Taking the difference in the radius of small intestine into consideration, the Fa values of these drugs in cynomolgus monkeys can be estimated to be comparable with those in human. This result might support the report of Chiou and Buehler in which the fraction of dose absorbed of 43 drugs in cynomolgus monkeys correlated well with those in humans. ${ }^{26)}$

The $P_{\text {eff }}$ value of verapamil was not plotted on the correlation line obtained for other drugs in Fig. 3. Verapamil is reported to be a typical substrate of P-glycoprotein (P-gp). ${ }^{27,28)}$ We have already demonstrated that the expression levels of mRNA for efflux transporters such as P-gp and breast cancer resistant protein (BCRP) in the intestine of cynomolgus monkeys were much higher than those in humans. ${ }^{3)}$ Therefore, the lower $P_{\text {eff }}$ value of verapamil can be attributed to the high activity of $\mathrm{P}$-gp, indicating a strong barrier function of the intestine of cynomolgus monkeys against the absorption of $\mathrm{P}$ gp substrate compounds.

Sandström et al. demonstrated that the $P_{\text {eff }}$ of verapamil increased due to saturation of P-gp when the verapamil concentrations in perfusate were increased from $4 \mathrm{mg} / 1$ to $40 \mathrm{mg} / \mathrm{l}$ in humans. ${ }^{29)}$ Since the verapamil concentration in the present study $(10 \mu \mathrm{M})$ is almost same as $4 \mathrm{mg} / \mathrm{l}$, the $P_{\text {eff }}$ value of verapamil in cynomolgus monkeys might be in- creased by increasing the concentration of verapamil in the perfusate. The further investigations with varying concentration of drugs and/or with specific P-gp inhibitor should be necessary to clarify the contribution of P-gp more detail.

Other than the efflux transporters, intestinal enzymes might be responsible for the low oral BA of drugs. Intestinal epithelial cells are reported to contain various enzymes related to phase I and phase II metabolism reactions. Various methods have been utilized to assess the intestinal drug metabolism. ${ }^{30)}$ Komura and Iwaki have reported the possibility of in vitro stability assay with intestinal microsomes to evaluate the species differences in the intestinal first-pass metabolism of CYP3A substrates. ${ }^{31)}$ Furthermore, kinetic models to predict the intestinal first-pass metabolism from in vitro experiments have been reported. ${ }^{32,33)}$

On the other hand, simultaneous perfusion method of the intestinal tract and the mesenteric vein have often been used to evaluate directly the intestinal first-pass metabolism in rats. ${ }^{5,6)}$ However, since the method requires complicated operations and is difficult to apply to the big animals such as monkeys, in this study, we have measured the concentration of drugs in both portal and peripheral veins during the intestinal perfusion to calculate $\mathrm{Fg}$ value. In our method, amount of the drug metabolized in the intestinal epithelial cells are calculated as the difference between the eliminated amount from the intestinal tract and the absorbed amount into the portal vein. Although the eliminated amount can be calculated easily from the intestinal perfusion data, calculation of the absorbed amount into the portal vein needs the data of portal and peripheral drug concentrations and the $V_{\text {portal }}$ (Eqs. $4,5)$.

The $V_{\text {portal }}$ value in cynomolgus monkeys was reported to be approximately $30 \mathrm{ml} / \mathrm{min} / \mathrm{kg} .{ }^{34)}$ However, because hepatic and portal blood flow is decreased under anesthetic condition, ${ }^{35,36)}$ the $V_{\text {portal }}$ values during the perfusion study are considered to be lower than the reported value. In this study, the $V_{\text {portal }}$ value in each monkey was obtained according to Eq. 6 by assuming that antipyrine is not metabolized in the intestine $(\mathrm{Fg}=1)$. Since we have already reported that $\mathrm{Fa}^{*} \mathrm{Fg}$ of antipyrine in cynomolgus monkey was 0.99 (also shown in Table 2), ${ }^{4)}$ it is reasonable to assume that the intestinal metabolism of antipyrine is negligible. In this study, the mean $V_{\text {portal }}$ value was calculated to be $14.2 \pm 8.4 \mathrm{ml} / \mathrm{min} / \mathrm{kg}$ that was almost half of the reported value in cynomolgus monkeys, suggesting the effect of anesthesia. Validity to use this value can be proved from the facts that 1) Fg values of acetaminophen and piroxicam obtained in this study were almost comparable with the results of the in vivo $\mathrm{PK}$ analysis, 2) Fg values of propranolol, verapamil and midazolam was enhanced to about 0.8 in the presence of ATB. In addition, by calculating the $V_{\text {portal }}$ values for each monkey, effect of interindividual difference in the portal blood flow on the Fg calculation of drugs tested as cassette study can be cancelled out.

As a result, Fg values of verapamil and midazolam were evaluated to be low (Table 2). In addition, Fg values of both drugs increased significantly in the presence of ATB. Metabolism of midazolam in the liver microsomes of cynomolgus monkeys is inhibited by the human anti-CYP3A antiserum, and coadministration of ketoconazole increased the AUC values of midazolam. ${ }^{37)}$ These data suggested that the metabo- 
lism of midazolam in cynomolgus monkeys is also dependent on the CYP3A enzyme. Therefore, midazolam was thought to be a reference of CYP3A substrate in cynomolgus monkeys. These results clearly pointed out the major impact of intestinal first-pass metabolism by CYP3A enzyme on the low oral BA in cynomolgus monkeys. Other than CYP3A substrates, propranolol and acetaminophen (substrates of CYP2D and UGT, respectively) were proved to undergo the first-pass metabolism in the intestine. Since $\mathrm{Fa} * \mathrm{Fg}$ values of both drugs in humans to be 1 (Table 2), ${ }^{4}$ these drugs are mainly metabolized by liver, but not in the intestine in humans. Therefore, species differences in the activity of intestinal enzymes were revealed between cynomolgus monkeys and humans.

In conclusion, an in situ intestinal perfusion method is revealed to be useful to quantitatively evaluate the $P_{\text {eff }}$ and $\mathrm{Fg}$ of drugs, simultaneously. From the results obtained in this study, it can be concluded that intestine of cynomolgus monkeys plays a critical role as a barrier against the absorption of various drugs those are substrates of efflux transporters and metabolizing enzymes, and that is one of the main causes of the low oral BA of drugs in cynomolgus monkeys.

Acknowledgements The authors gratefully acknowledge Mr. Shunichi Kitajima of Primate Ltd. for his technical assistance to perform the experiments.

\section{REFERENCES}

1) Sakuda S., Akabane T., Teramura T., Xenobiotica, 36, 331-340 (2006).

2) Nishimura T., Amano N., Kubo Y., Ono M., Kato Y., Fujita H., Kimura Y., Tsuji A., Drug Metab. Dispos., 35, 1275-1284 (2007).

3) Takahashi M., Washio T., Suzuki N., Igeta K., Fujii Y., Hayashi M., Shirasaka Y., Yamashita S., Mol. Pharm., 5, 340-348 (2008).

4) Takahashi M., Washio T., Suzuki N., Igeta K., Yamashita S., J. Pharm. Sci., 98, 4343-4353 (2009).

5) Tamura S., Tokunaga Y., Ibuki R., Amidon G. L., Sezaki H., Yamashita, S., J. Pharmacol. Exp. Ther, 306, 310-316 (2003).

6) Mihara K., Matsumura M., Yoshioka E., Hanada K., Nakasa H., Ohmori S., Kitada M., Ogata H., Pharm. Res., 18, 1131-1137 (2001).

7) Kasim N. A., Whitehouse M., Ramachandran C., Bermejo M., Lennernäs H., Hussain A. S., Junginger H. E., Stavchansky S. A., Midha K. K., Shah V. P., Amidon, G. L., Mol. Pharm., 1, 85-96 (2004).

8) Balimane P. V., Chong S., Morrison R. A., J. Pharmacol. Toxicol. Methods, 44, 301-312 (2000).
9) Hidalgo I. J., Curr. Top. Med. Chem., 1, 385-401 (2001).

10) Hidalgo I. J., Raub T. J., Borchardt R. T., Gastroenterology, 96, 736749 (1989).

11) Artursson P., Karlsson J., Biochem. Biophys. Res. Commun., 175, $880-885$ (1991)

12) Yamashita S., Furubayashi T., Kataoka M.. Sakane T., Sezaki H., Tokuda H., Eur. J. Pharm. Sci., 10, 195-204 (2000).

13) Yee S., Pharm. Res., 14, 763-766 (1997).

14) Irvine J. D., Takahashi L., Lockhart K., Cheong J., Tolan J. W., Selick H. E., Grove J. R., J. Pharm. Sci., 88, 28-33 (1999).

15) Volpe D. A., J. Pharm. Sci., 97, 712-725 (2008).

16) Sugano K., Hamada H., Machida M., Ushio H., Saitoh K., Terada K., Int. J. Pharm., 228, 181-188 (2001).

17) Avdeef A., Bendels S., Faller B., Kansy M., Sugano K., Yamauchi Y., J. Pharm. Sci., 96, 2893-2909 (2007).

18) Fagerholm U., Johansson M., Linnernäs H., Pharm. Res., 13, 13361342 (1996).

19) Linnernäs H., J. Pharm. Sci., 87, 403-410 (1998).

20) Takamatsu N., Welage L. S., Idkaidek N. M., Liu D. Y., Lee P. I., Hayashi Y., Rhie J. K., Linnernäs H., Barnett J. L., Shah V. P., Lesko L., Amidon G. L., Pharm. Res., 14, 1127-1132 (1997).

21) Pang K. S., Cherry W. F. Ulm E. H., J. Pharmacol. Exp. Ther, 233, $788-795$ (1985)

22) Iwao T., Inoue K., Hayashi Y., Yuasa H., Watanabe J., Drug Metab. Pharmacokinet., 17, 546-553 (2002).

23) Ungell A. L., Nylander S., Bergstrand S., Sjöberg Å., Linnernäs H., J. Pharm. Sci., 87, 360-366 (1998).

24) Paine M. F., Khalighi M., Fisher J. M., Shen D. D., Hunze K. L., Marsh C. L., Perkins J. D., Thummel K. E., J. Pharmacol. Exp. Ther., 283, 1552-1562 (1997).

25) Kararli T. T., Biopharm. Drug Dispos., 16, 351-380 (1995).

26) Chiou W. L., Buehler P. W., Pharm. Res., 19, 868-874 (2002).

27) Kroemer H. K., Gautier J. C., Beaune P., Henderson C., Wolf C. R., Eichelbaum M., Naunyn. Schmiedebergs Arch. Pharmacol., 348, 332-337 (1993).

28) Busse D., Cosme J., Beaune P., Kroemer H. K., Eichelbaum M., Naunyn. Schmiedebergs Arch. Pharmacol., 353, 116-121 (1995).

29) Sandström R., Karlsson A., Knutson L., Linnernäs H., Pharm. Res., 15, 856-862 (1998).

30) van de Kerkhof E. G., de Graaf I. A., Groothuis M. M., Curr. Drug Metab., 8, 658-675 (2007).

31) Komura H., Iwaki M., J. Pharm. Sci., 97, 1775-1800 (2008).

32) Yang J., Jamei M., Yeo K. R., Tucker G. T., Rostami-Hodjegan A., Curr. Drug Metab., 8, 676-684 (2007)

33) Kato M., Drug Metab. Pharmacokinet., 23, 87-94 (2008).

34) Davies B., Morris T., Pharm. Res., 10, 1093-1095 (1993).

35) Uhing M. R., Beno D. W., Jiyamapa-Serna V. A., Chen Y., Galinsky R. E., Hall S. D., Kimura R. E., Drug Metab. Dispos., 32, 1325-1330 (2004)

36) Reilly C. S., Merrell J., Wood A. J., Koshakji R. P., Wood M., Br. J. Anaesth., 60, 791-796 (1988).

37) Kanazu T., Yamaguchi Y., Okamura N., Baba T., Koike M., Xenobioteca, 34, 391-402 (2004). 